\title{
Birds of Sabaragamuwa University campus, Buttala, Sri Lanka
}

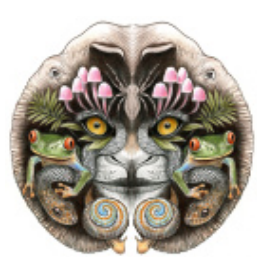

\author{
Thilina Dilan Surasinghe ${ }^{1}$ \& Chamitha De Alwis ${ }^{2}$
}

${ }^{1}$ Teaching Assistant in Biological Sciences, Graduate Student in Wildlife and Fisheries Biology, Department of Forestry and Natural Resources, 261, Lehotsky Hall, Clemson University, Clemson, SC 29634-0317, USA

${ }^{2}$ Department of Natural Resources, Sabaragamuwa University of Sri Lanka, Buttala, Sri Lanka

Email: ${ }^{1}$ tsurasi@clemson.edu

Date of publication (online): 26 May 2010 Date of publication (print): 26 May 2010 ISSN 0974-7907 (online) | 0974-7893 (print)

Editor: Rajah Jayapal

\section{Manuscript details:}

Ms \# o2113

Received 28 December 2008

Final revised received 12 March 2010

Finally accepted 09 April 2010

Citation: Surasinghe, T.D. \& C. De Alwis (2010). Birds of Sabaragamuwa University campus, Buttala, Sri Lanka. Journal of Threatened Taxa 2(5): 876-888

Copyright: (c) Thilina Dilan Surasinghe \& Chamitha De Alwis 2010. Creative Commons Attribution 3.0 Unported License. JoTT allows unrestricted use of this article in any medium for non-profit purposes, reproduction and distribution by providing adequate credit to the authors and the source of publication.

Author Details: THILINA DILAN SURASINGHE has conducted a significant number of research on biodiversity of Sri Lanka and published several peer-reviewed scientific articles in numerous journals. He has served as an junior faculty in several Sri Lankan public universities. Currently, he is reading a PhD, majoring Wildlife Biology. His dissertation work is on diversity and distribution of stream salamanders in the montane temperate areas.

Chamitha De Alwis is well experienced and has good knowledge about biodiversity of Sri Lanka. $\mathrm{He}$ has worked in multiple research projects on ecological and conservation related research in Sri Lanka.

Author Contribution: TDS contributed in formulating the idea of the research, writing and structuring the paper, planning the field procedures, analyzing and presenting the data, literature review on the topic. CDA contributed in conducting and coordinating field research, formulating and executing the field procedure.

Acknowledgements: We would like to express our gratitude to the undergraduate students of the Sabaragamuwa University Campus for providing their support during the fieldwork and Ms. Enoka Kudavidanage for her valuable insights during this research.

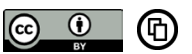

OPEN ACCESS | FREE DOWNLOAD
Abstract: We conducted a bird survey in the Sabaragamuwa University premises in southeastern Sri Lanka between 2001 and 2004. We recorded 145 bird species, representing 17 orders and 51 families from the campus. The birdlife included Redfaced Malkoha, a globally Vulnerable species and four Near Threatened taxa. The university premises suffer from severe habitat alteration largely owing to fire, filling-up of aquatic habitats, resource over-extraction, improper waste management, invasion by exotic species and livestock grazing. Several conservation measures, including habitat management strategies such as restoration of riparian vegetation, and wetlands, increasing plant diversity in home gardens and prevention of secondary successions in grasslands are recommended to protect the campus environment and to conserve its avifaunal diversity.

Keywords: Birds, conservation, habitat management, Sabaragamuwa University, Sri Lanka

\section{INTRODUCTION}

The Indian Ocean island of Sri Lanka $\left(65610 \mathrm{~km}^{2}\right)$ is rich in avifaunal diversity. Over 471 species of birds representing 20 orders and 76 families have been recorded in Sri Lanka, (Kotagama et al. 2006). These include 225 breeding residents, 128 winter visitors, four summer visitors, 106 vagrants, and two passage migrants (Rasmussen \& Anderton 2005). Of them, 33 are endemic to the island (Kotagama et al. 2006). Owing to this high diversity and endemism, Sri Lanka has been recognized as a country with "Important Bird Areas", a "key Asian region for threatened birds" and an "Endemic Bird Area" (Kaluthota \& Kotagama 2005). Therefore, it is imperative to document distribution, habitat association, threats and conservation measures on avifauna.

Extensive studies on ecology and distribution of birds of Sri Lanka have so far been conducted in and around protected areas in the wet and dry zones of Sri Lanka. Studies on avifauna of the intermediate bioclimatic zone and landscapes outside protected areas are very scarce. Several such sites outside protected areas yet remain undocumented leading to gaps in knowledge of distribution of the island's avifauna. Further, the role of humanaltered landscapes in conservation of birds has been greatly neglected. In the face of rapid economic development and increasing human population, the extent of conservation lands is gradually reducing. It is essential to study the suitability of semi-natural landscapes such as human settlements in rural areas for the long-term survival of native biodiversity (Hietala-Koivu et al. 2004).

With this rationale, we surveyed the premises of the Sabaragamuwa University of Sri Lanka in Buttala to study diversity and habitat associations of birds. We also studied threats on birds and then recommended conservation and management actions that would enhance the avifaunal diversity of the site, while continuing with innocuous human activities and habitation. 


\section{Study site}

The study site is situated in a rural village of the southeastern Sri Lanka, (6046'28.32"N-81015'28.58"E and 6 $\left.66^{\prime} 3.54^{\prime \prime} \mathrm{N}-31^{0} 15^{\prime} 35.65^{\prime \prime} \mathrm{E}\right)$. Our study area was originally a homestead, comprising home gardens, private lands and a teak plantation (De Alwis \& Surasinghe 2006). In 1988, the site was the focus of a government sponsored rural area development scheme that altered the original condition of the area due to heavy anthropogenic disturbance. In the year 1993, the Sabaragamuwa University of Sri Lanka established the Faculty of Applied Sciences in this land area, with significant changes in the land-use and land-cover structure after the rural development program. Since then, the vegetated area of the university premises did not receive any major spatiallyextensive disturbances. However, local disturbances continue to prevail in different frequencies and different intensities (De Alwis \& Surasinghe 2006). Currently, the university premises consist of rocky grasslands, dry-mixed semi-evergreen forests, scrublands, residential areas, home gardens and wetlands. These wetlands include lotic habitats such as perennial streams and seasonal creeks and lentic habitats such as seasonal pools and semi-permanent man-made ponds (Image 1) (De Alwis et al. 2006). The university premises are 125 acres in area. Thirty percent of the premises are covered scrublands, with $40 \%$ of the area covered by residential areas and home gardens. Both rocky grasslands and dry-mixed semi-evergreen forests occupy a similar area of extent accounting for $30 \%$ of the whole area. The overall region comprises forest fragments, grasslands, scrublands, riverine forests and marshlands, each habitat vary in size. Further, there are large extents of home gardens and agricultural lands cultivated with annual crops such as vegetables and tobacco in this region.

In terms of geo-climatic regionalization, the site falls under the lowland (elevation less than $300 \mathrm{~m}$ in average) dry intermediate zone of the island. The

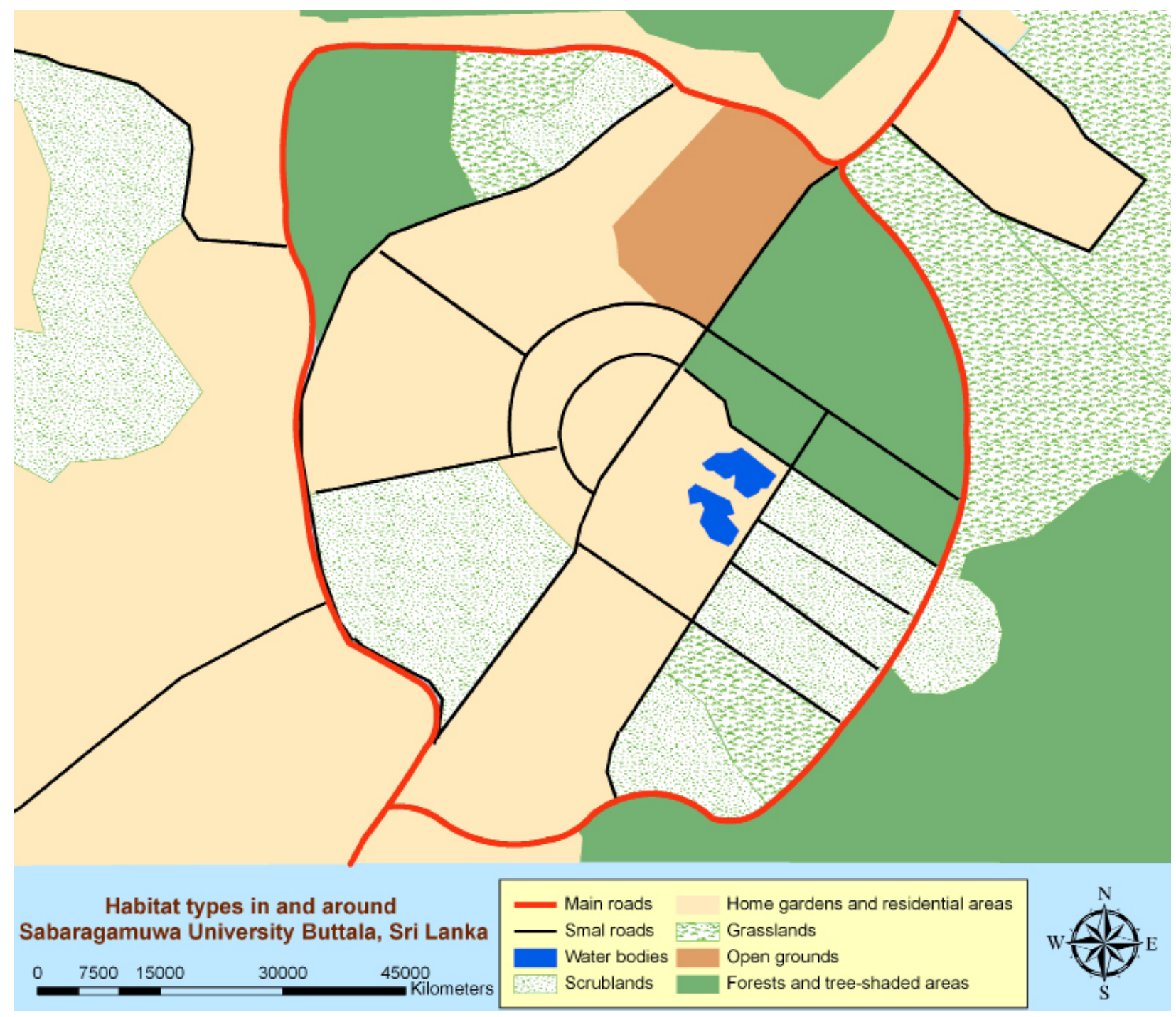

Image 1. A map of the study site - the Sabaragamuwa University premises 


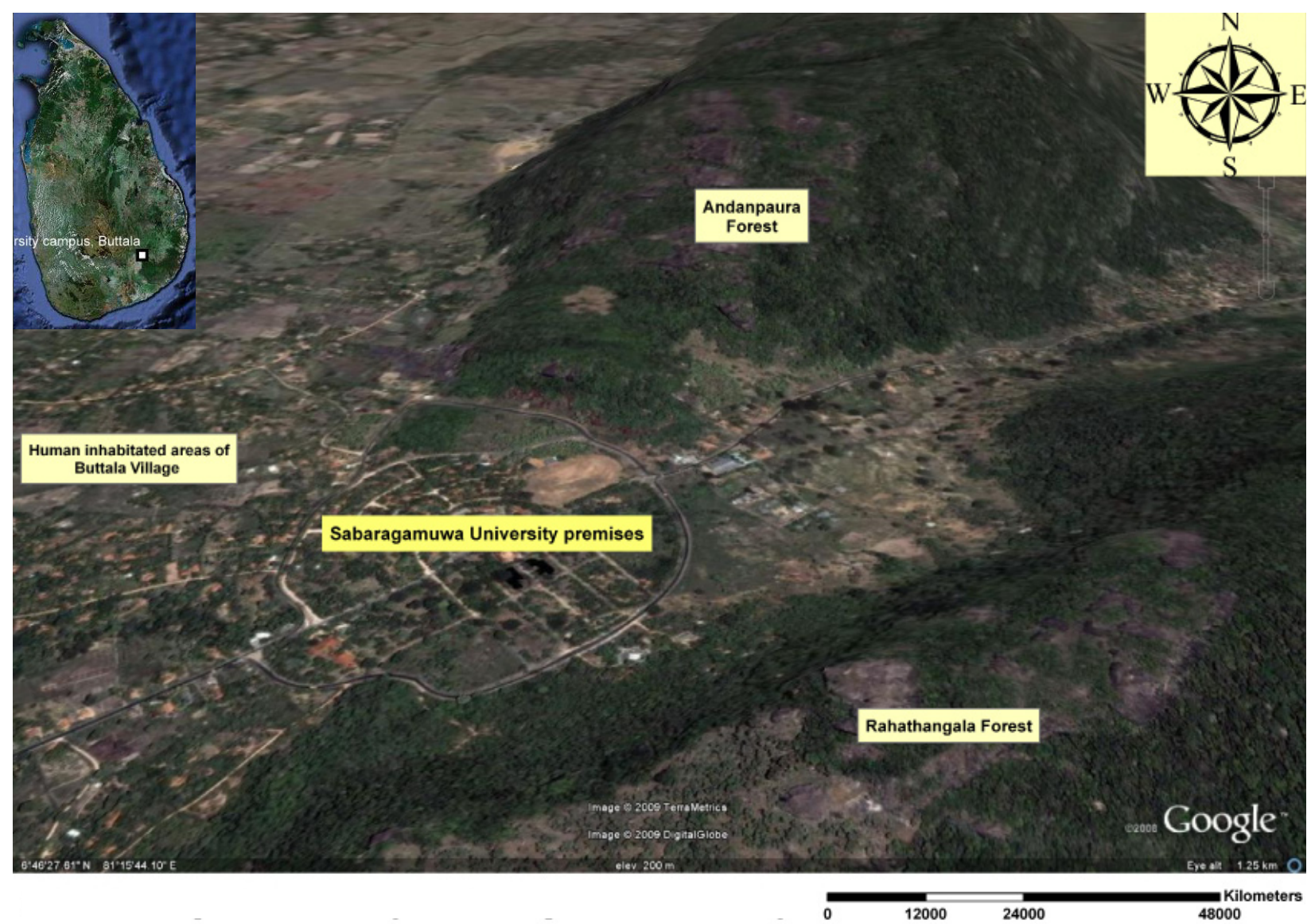

Image 2. A satellite image of the study site - the Sabaragamuwa University premises

annual precipitation ranges between $900-1500 \mathrm{~mm}$ and the annual average temperature is approximately $27^{\circ} \mathrm{C}$ (Survey Department 1988). Floristically, the university premises fall within the tropical moist semi-evergreen forests and savannah forests (Aston \& Gunetilleke 1987a, 1987b). Although, the terrain of the entire region can be considered as undulating, the university premises are relatively flat. The study area, topographically and ecologically is a habitat island which lies in close proximity to two relatively large hilly forest fragments; "Andampahura" forest and "Rahathangala" forest. The university premises are separated from the two fragments mentioned above by the Colombo - Potuvil highway (Image 2) (Survey Department 1987). Following are some of the flowering plants recorded in this region: Melia dubia, Mangifera zeylanica, Pometia eximia, Artocarpus nobilis, Filicium decipiens, Turpinia malabarica, Rejoua dichotoma, Anamirta cocculus, Artabotrys uncinatus, Paramignya monophylla, Anodendron manubriatum, Entada spp., Pothos scandens Micromelum ceylanicum, Goniothalamus spp., Dracaena thwaitesii, Ophiorrhiza mungos.

\section{METHODOLOGY}

The bird survey in the university premises was done for four years from 2001 to 2004 . The survey techniques used included visual encounter surveys, line transects, spot counts and mist net trapping covering all the habitat types within the study site, as outlined above. The survey was done during various time periods of the day using binoculars, monocular telescopes and direct observations. In order to consider both diurnal and nocturnal species, the survey was conducted in three sessions: 0600-1200 hr, 1600-1900 hr and 2100-2300 hr. Surveying in three different time frames of the day adequately sample the realized temporal niche of the birds in the university premises. The avifaunal species richness of six distinct habitat types (grasslands, open grounds, dry-mixed semi-evergreen forests, scrub forests, residential areas and home gardens, limnotic habitats) within the university premises is recorded. For the purpose of identification of birds, popular field guilds like Harrison (1999) and Kotagama \& Wijesinghe (1998) were used. During the bird surveys, human activities that potentially pose threats to the bird populations were also noted. 


\section{RESULTS AND ANALYSIS}

During the survey, a total of 145 bird species including 15 Sri Lankan endemics were recorded in the study period, representing 17 orders and 51 families. This would make up nearly $31 \%$ of the Sri Lanka's total avifaunal diversity. Among them, 15 species were winter visitors and 122 were breeding residents.

Our results showed that the residential areas including home gardens harboured the highest number of species representing $68 \%$ of the all the species recorded in the survey (Fig. 1). Although the limnotic habitats had the lowest species richness accounting only for $12 \%$ of the total avifaunal diversity of the study area, most species recorded in limnotic habitats were restricted to such aquatic habitats. The details of habitat occupancy are shown in the Table 1. Further analysis on habitat association revealed that 32 species of birds (forming $22 \%$ ) were habitat specialists as they were recorded from only one habitat type. For instance, White-necked Stork, Common Sandpiper, Stork-billed Kingfisher and Small Kingfisher were restricted to limnotic habitats. Similarly, Sri Lankan Lorikeet, Blossom-headed Parakeet, Layard's Parakeet were only recorded from the home gardens. The Racket-tailed Drongo, Black-capped Bulbul, Whiterumped Sharma and Layard's Flycatcher were limited to the dry-mixed semi-evergreen forests. The Indian Pipit was only observed in open grounds. Blyth's Reed Warbler and Great Reed Warbler were some of the species that were seemingly confined to grasslands. Interestingly, the number of birds that occupied all five habitat types in the campus premises was significantly low (5 species). On the contrary, the number of bird species that occupied only one type of habitat was significantly high (32 species). Thirty percent of the species recorded in this study associated two habitat types within the campus premises.

\section{DISCUSSION}

The diversity of birds (Table 1) and their distribution with respect to available habitat types show the importance of the university premises as an ideal bird habitat, within the intermediate zone of Sri Lanka. Five birds recorded in this study are listed in the Global Red Data Book (IUCN 2009). Among them, one species (Red-faced Malkoha) is Vulnerable and four are Near Threatened (Spot-billed Pelican, Malabar Pied Hornbill, White Ibis, and Painted Stork). Besides, 12 species are considered nationally threatened according to the 2007 List of Threatened Fauna and Flora of Sri Lanka (IUCNSL \& Ministry of Environment and Natural Resources 2008). The high preference of birds to this site can be attributed to many factors:

1. The university premises consist of a mosaic of habitats. Grasslands, open grounds, dry-mixed semievergreen forests, scrub forests, home gardens and limonitic habitats are the major habitats. Riparian vegetation, road verges and small Teak estates are minor habitats. Habitat heterogeneity favors habitat specialists (through niche partitioning) and birds with broad niches.

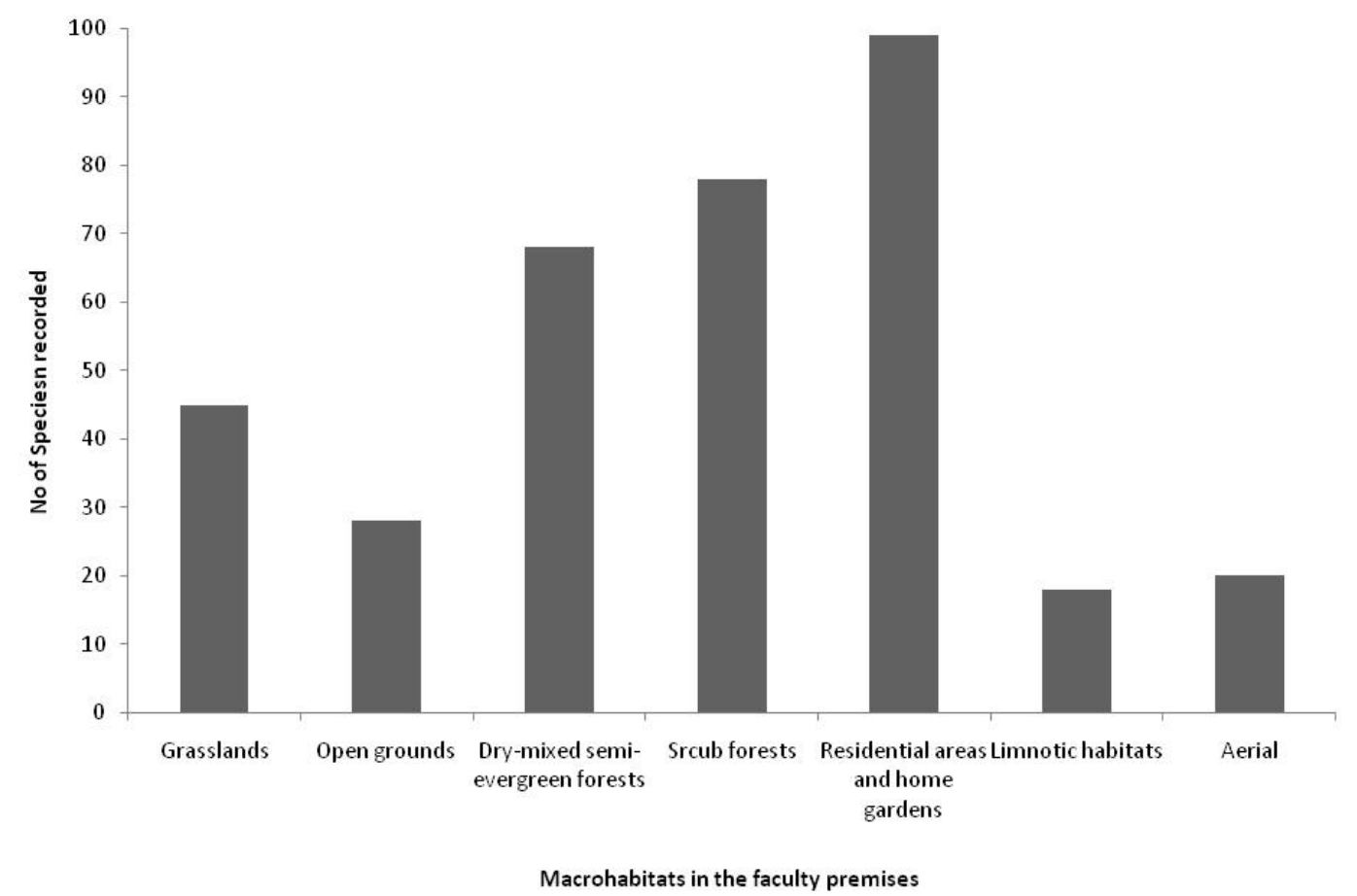

Figure 1. Number of bird species recorded from each habitat type in the Sabaragamuwa University premises 
Table 1. Different habitats (grasslands, open grounds, dry-mixed semi-evergreen forests, scrub forests, residential areas and home gardens, limnotic habitats, aerial) occupied by the bird species recorded within the premises.

\begin{tabular}{|c|c|c|c|c|c|c|c|c|c|}
\hline \multirow[b]{2}{*}{ Species } & \multirow[b]{2}{*}{ 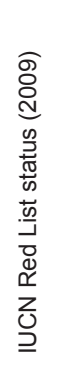 } & \multirow[b]{2}{*}{ 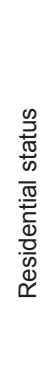 } & \multicolumn{6}{|c|}{ Habitat types } & \multirow[b]{2}{*}{$\frac{\pi}{2}$} \\
\hline & & & $\begin{array}{l}\frac{0}{0} \\
\frac{1}{0} \\
\frac{\pi}{w} \\
0 \\
\mathbb{0} \\
0 \\
0\end{array}$ & 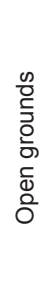 & 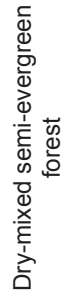 & 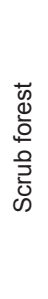 & 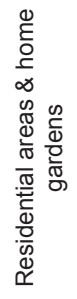 & 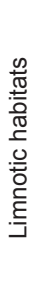 & \\
\hline \multicolumn{10}{|l|}{$\begin{array}{l}\text { Order Pelecaniformes } \\
\text { Family Pelecanidae }\end{array}$} \\
\hline $\begin{array}{l}\text { Spot-billed Pelican } \\
\text { Pelecanus philippensis }\end{array}$ & NT & $\mathrm{br}$ & & & & & & & + \\
\hline \multicolumn{10}{|l|}{ Family Phalacrocoracidae } \\
\hline $\begin{array}{l}\text { Little Cormorant } \\
\text { Phalacrocorax niger }\end{array}$ & & br & & & & & & + & + \\
\hline $\begin{array}{l}\text { Indian Shag } \\
\text { Phalacrocorax fuscicollis }\end{array}$ & & $\mathrm{br}$ & & & & & & + & + \\
\hline \multicolumn{10}{|l|}{$\begin{array}{l}\text { Order Ciconiiformes } \\
\text { Family Ardeidae }\end{array}$} \\
\hline $\begin{array}{l}\text { Little Egret } \\
\text { Egretta garzetta }\end{array}$ & & $\mathrm{br}$ & & & & & & + & + \\
\hline $\begin{array}{l}\text { Grey Heron } \\
\text { Ardea cinerea }\end{array}$ & & $\mathrm{br}$ & & & & & & & + \\
\hline $\begin{array}{l}\text { Purple Heron } \\
\text { Ardea purpurea }\end{array}$ & & $\mathrm{br}$ & & & & & & + & + \\
\hline $\begin{array}{l}\text { Median Egret } \\
\text { Mesophoyx intermedia }\end{array}$ & & $\mathrm{br}$ & & & & & & + & + \\
\hline $\begin{array}{l}\text { Cattle Egret } \\
\text { Bubulcus ibis }\end{array}$ & & $\mathrm{br}$ & + & + & & + & + & + & \\
\hline $\begin{array}{l}\text { Indian Pond Heron } \\
\text { Ardeola grayii }\end{array}$ & & $\mathrm{br}$ & + & + & & + & + & + & \\
\hline $\begin{array}{l}\text { Great Egret } \\
\text { Casmerodius albus }\end{array}$ & & $\mathrm{br}$ & & & & & & + & + \\
\hline \multicolumn{10}{|l|}{ Family Ciconiidae } \\
\hline $\begin{array}{l}\text { Painted Stork } \\
\text { Mycteria leucocephala }\end{array}$ & NT & $\mathrm{br}$ & & & & & & & + \\
\hline $\begin{array}{l}\text { White-necked Stork } \\
\text { Ciconia episcopus }\end{array}$ & & $\mathrm{br}$ & & & & & + & & + \\
\hline $\begin{array}{l}\text { Asian Open Bill } \\
\text { Anastomus oscitans }\end{array}$ & & $\mathrm{br}$ & & & & & & & + \\
\hline \multicolumn{10}{|l|}{ Family Threskiornithidae } \\
\hline $\begin{array}{l}\text { White Ibis } \\
\text { Threskiornis melanocephalus }\end{array}$ & NT & br & & & & & & & + \\
\hline \multicolumn{10}{|l|}{$\begin{array}{l}\text { Order Anseriformes } \\
\text { Family Dendrocygnidae }\end{array}$} \\
\hline $\begin{array}{l}\text { Lesser Whistling Duck } \\
\text { Dendrocygna javanica }\end{array}$ & & $\mathrm{br}$ & & & & & & & + \\
\hline
\end{tabular}




\begin{tabular}{|c|c|c|c|c|c|c|c|c|}
\hline \multicolumn{9}{|l|}{$\begin{array}{l}\text { Order Falconiformes } \\
\text { Family Accipitridae }\end{array}$} \\
\hline $\begin{array}{l}\text { Crested Honey-Buzzard } \\
\text { Pernis ptilorhyncus }\end{array}$ & $\mathrm{br}$ & & & + & + & + & & \\
\hline $\begin{array}{l}\text { Brahminy Kite } \\
\text { Haliastur Indus }\end{array}$ & $\mathrm{br}$ & & & & & + & & + \\
\hline $\begin{array}{l}\text { Crested Serpent Eagle } \\
\text { Spilornis cheela }\end{array}$ & $\mathrm{br}$ & & & + & + & + & & \\
\hline $\begin{array}{l}\text { Shikra } \\
\text { Accipiter badius }\end{array}$ & $\mathrm{br}$ & & & + & + & + & & \\
\hline $\begin{array}{l}\text { Besra Sparrow Hawk } \\
\text { Accipiter virgatus }\end{array}$ & br & & & + & & + & & \\
\hline $\begin{array}{l}\text { Common Buzzard } \\
\text { Buteo buteo }\end{array}$ & wV & & & & & & & + \\
\hline $\begin{array}{l}\text { Black Eagle } \\
\text { Ictinaetus malayensis }\end{array}$ & $\mathrm{br}$ & & & + & & + & & \\
\hline $\begin{array}{l}\text { Crested Hawk Eagle } \\
\text { Spizaetus cirrhatus }\end{array}$ & $\mathrm{br}$ & & & & + & + & & \\
\hline \multicolumn{9}{|l|}{ Family Falconidae } \\
\hline $\begin{array}{l}\text { Peregrine Falcon } \\
\text { Falco peregrinus }\end{array}$ & br & & & + & & + & & \\
\hline \multicolumn{9}{|l|}{$\begin{array}{l}\text { Order Galliformes } \\
\text { Family Phasianidae }\end{array}$} \\
\hline $\begin{array}{l}\text { Sri Lanka Spurfowl } \\
\text { Galloperdix bicalcarata }\end{array}$ & brE & + & & + & + & & & \\
\hline $\begin{array}{l}\text { Sri Lanka Junglefowl } \\
\text { Gallus lafayettii }\end{array}$ & brE & + & & + & + & & & \\
\hline $\begin{array}{l}\text { Indian Peafowl } \\
\text { Pavo cristatus }\end{array}$ & $\mathrm{br}$ & + & + & & + & + & & \\
\hline \multicolumn{9}{|l|}{$\begin{array}{l}\text { Order Turniciformes } \\
\text { Family Turnicidae }\end{array}$} \\
\hline $\begin{array}{l}\text { Barred Bustard-Quail } \\
\text { Turnix suscitator }\end{array}$ & $\mathrm{br}$ & + & + & & + & & & \\
\hline \multicolumn{9}{|l|}{$\begin{array}{l}\text { Order Gruiformes } \\
\text { Family Rallidae }\end{array}$} \\
\hline $\begin{array}{l}\text { White-breasted Waterhen } \\
\text { Amaurornis phoenicurus }\end{array}$ & $\mathrm{br}$ & & & & & & + & \\
\hline \multicolumn{9}{|l|}{$\begin{array}{l}\text { Order Charadriiformes } \\
\text { Family Charadriidae }\end{array}$} \\
\hline $\begin{array}{l}\text { Yellow-wattled Lapwing } \\
\text { Vanellus malarbaricus }\end{array}$ & br & & + & & & & & \\
\hline $\begin{array}{l}\text { Red-wattled Lapwing } \\
\text { Vanellus indicus }\end{array}$ & $b r$ & + & + & & & & + & \\
\hline \multicolumn{9}{|l|}{ Family Scolopacidae } \\
\hline $\begin{array}{l}\text { Common Sandpiper } \\
\text { Actitis hypoleucos }\end{array}$ & wv & & & & & & + & \\
\hline \multicolumn{9}{|l|}{$\begin{array}{l}\text { Order Columbiformes } \\
\text { Family Columbidae }\end{array}$} \\
\hline $\begin{array}{l}\text { Spotted Dove } \\
\text { Streptopelia chinensis }\end{array}$ & $\mathrm{br}$ & + & + & + & + & + & & \\
\hline $\begin{array}{l}\text { Emerald Dove } \\
\text { Chalcophaps indica }\end{array}$ & $\mathrm{br}$ & & + & & & + & & \\
\hline
\end{tabular}




\begin{tabular}{|c|c|c|c|c|c|c|c|c|}
\hline $\begin{array}{l}\text { Orange-breasted Green Pigeon } \\
\text { Treron bicincta }\end{array}$ & & $\mathrm{br}$ & & & + & & + & \\
\hline $\begin{array}{l}\text { Pompadour Green Pigeon } \\
\text { Treron pompadora }\end{array}$ & & brE & & & + & & + & \\
\hline $\begin{array}{l}\text { Green Imperial Pigeon } \\
\text { Ducula aenea }\end{array}$ & & $\mathrm{br}$ & & & + & & + & \\
\hline \multicolumn{9}{|l|}{$\begin{array}{l}\text { Order Psittaciformes } \\
\text { Family Psittacidae }\end{array}$} \\
\hline $\begin{array}{l}\text { Alexandrine Parakeet } \\
\text { Psittacula eupatria }\end{array}$ & & $\mathrm{br}$ & & & + & & + & + \\
\hline $\begin{array}{l}\text { Rose-ringed Parakeet } \\
\text { Psittacula krameri }\end{array}$ & & $\mathrm{br}$ & & & + & & + & + \\
\hline $\begin{array}{l}\text { Sri Lankan Lorikeet } \\
\text { Loriculus beryllinus }\end{array}$ & & $\mathrm{brE}$ & & & & & + & \\
\hline $\begin{array}{l}\text { Blossom-headed Parakeet } \\
\text { Psittacula cyanocephala }\end{array}$ & & br & & & & & + & + \\
\hline $\begin{array}{l}\text { Layard's Parakeet } \\
\text { Psittacula calthropae }\end{array}$ & & $\mathrm{brE}$ & & & & & + & + \\
\hline \multicolumn{9}{|l|}{$\begin{array}{l}\text { Order Cuculiformes } \\
\text { Family Cuculidae }\end{array}$} \\
\hline $\begin{array}{l}\text { Pied Crested Cucko } \\
\text { Oxylophus jacobinus }\end{array}$ & & $\mathrm{br}$ & + & & & + & + & \\
\hline $\begin{array}{l}\text { Common Hawk Cuckoo } \\
\text { Cuculus varius }\end{array}$ & & $\mathrm{br}$ & & & + & & + & \\
\hline $\begin{array}{l}\text { Bay-banded Cuckoo } \\
\text { Cacomantis sonneratii }\end{array}$ & & $\mathrm{br}$ & & & & + & + & \\
\hline $\begin{array}{l}\text { Indian Plantive Cuckoo } \\
\text { Cacomantis passerinus }\end{array}$ & & wV & & & & + & + & \\
\hline $\begin{array}{l}\text { Asian Koel } \\
\text { Eudynamus scolopacea }\end{array}$ & & $\mathrm{br}$ & & & + & + & + & \\
\hline $\begin{array}{l}\text { Blue-faced Malkoha } \\
\text { Phaenicophaeus viridirostris }\end{array}$ & & br & + & & + & + & + & \\
\hline $\begin{array}{l}\text { Sirkeer Malkoha } \\
\text { Phaenicophaeus leschenaultii }\end{array}$ & & $\mathrm{br}$ & + & & & + & + & \\
\hline $\begin{array}{l}\text { Red-faced Malkoha } \\
\text { Phaenicophaeus pyrrhocephalus }\end{array}$ & VU & brE & & & & & + & \\
\hline \multicolumn{9}{|l|}{ Family Centropodidae } \\
\hline $\begin{array}{l}\text { Common Coucal } \\
\text { Centropus sinensis }\end{array}$ & & $\mathrm{br}$ & + & + & & + & + & \\
\hline \multicolumn{9}{|l|}{$\begin{array}{l}\text { Order Strigiformes } \\
\text { Family Strigidae }\end{array}$} \\
\hline $\begin{array}{l}\text { Collared Scops Owl } \\
\text { Otus bakkamoena }\end{array}$ & & $\mathrm{br}$ & & & + & + & + & \\
\hline $\begin{array}{l}\text { Forest Eagle Owl } \\
\text { Bubo nipalensis }\end{array}$ & & $\mathrm{br}$ & & & & + & + & \\
\hline $\begin{array}{l}\text { Brown Wood Owl } \\
\text { Strix leptogrammica }\end{array}$ & & $\mathrm{br}$ & & & + & & + & \\
\hline $\begin{array}{l}\text { Jungle Owlet } \\
\text { Glaucidium radiatum }\end{array}$ & & $\mathrm{br}$ & & & + & + & + & \\
\hline \multicolumn{9}{|l|}{ Family Caprimulgidae } \\
\hline $\begin{array}{l}\text { Common Indian Nightjar } \\
\text { Caprimulgus asiaticus }\end{array}$ & & $\mathrm{br}$ & + & + & & + & & \\
\hline
\end{tabular}




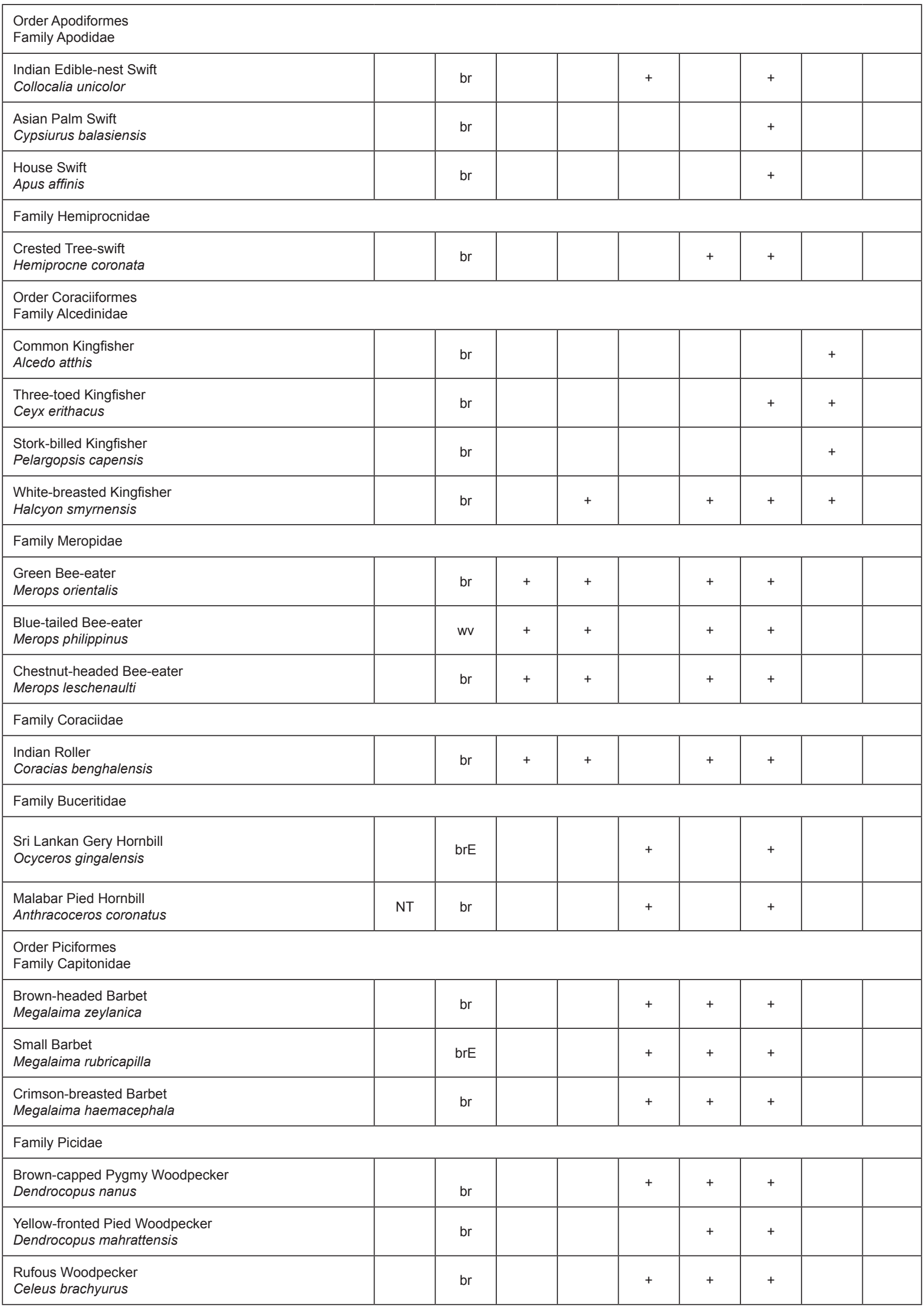




\begin{tabular}{|c|c|c|c|c|c|c|c|c|}
\hline $\begin{array}{l}\text { Red-backed Woodpecker } \\
\text { Dinopium benghalense psarodes }\end{array}$ & $\mathrm{br}$ & & & + & + & + & & \\
\hline $\begin{array}{l}\text { Crimson-backed Woodpecker } \\
\text { Chrysocolaptes lucidus }\end{array}$ & brE & & & + & + & + & & \\
\hline \multicolumn{9}{|l|}{$\begin{array}{l}\text { Order Passeriformes } \\
\text { Family Pittidae }\end{array}$} \\
\hline $\begin{array}{l}\text { Indian Pitta } \\
\text { Pitta brachyura }\end{array}$ & wV & + & & + & + & + & & \\
\hline \multicolumn{9}{|l|}{ Family Alaudidae } \\
\hline $\begin{array}{l}\text { Jerdon's Bushlark } \\
\text { Mirafra affinis }\end{array}$ & br & + & + & & & & & \\
\hline $\begin{array}{l}\text { Ashy-crowned Finch Lark } \\
\text { Eremopterix grisea }\end{array}$ & br & & + & & & & & \\
\hline $\begin{array}{l}\text { Oriental Skylark } \\
\text { Alauda gulgula }\end{array}$ & $\mathrm{br}$ & + & + & & & & & \\
\hline \multicolumn{9}{|l|}{ Family Hirundinidae } \\
\hline $\begin{array}{l}\text { Barn Swallow } \\
\text { Hirundo rustica }\end{array}$ & br & + & + & & + & + & & \\
\hline $\begin{array}{l}\text { Red-rumped Swallow } \\
\text { Hirundo daurica }\end{array}$ & brE & & + & & + & + & + & \\
\hline \multicolumn{9}{|l|}{ Family Laniidae } \\
\hline $\begin{array}{l}\text { Brown Shrike } \\
\text { Lanius cristatus cristatus }\end{array}$ & wV & + & & + & + & + & & \\
\hline $\begin{array}{l}\text { Philipppine Shrike } \\
\text { Lanius cristatus lucionensis }\end{array}$ & wV & & & & & & & \\
\hline \multicolumn{9}{|l|}{ Family Oriolidae } \\
\hline $\begin{array}{l}\text { Black-headed Oriole } \\
\text { Oriolus xanthornus }\end{array}$ & $\mathrm{br}$ & & & + & & + & & \\
\hline \multicolumn{9}{|l|}{ Family Dicruridae } \\
\hline $\begin{array}{l}\text { White-vented Drongo } \\
\text { Dicrurus caerulescens }\end{array}$ & $\mathrm{br}$ & + & & + & + & + & & \\
\hline $\begin{array}{l}\text { Greater Racket-tailed Drongo } \\
\text { Dicrurus andamanensis }\end{array}$ & $\mathrm{br}$ & & & + & & & & \\
\hline \multicolumn{9}{|l|}{ Family Artamidae } \\
\hline $\begin{array}{l}\text { Ashy Swallow-shrike } \\
\text { Artamus fuscus }\end{array}$ & $\mathrm{br}$ & + & + & & & & & \\
\hline \multicolumn{9}{|l|}{ Family Sturnidae } \\
\hline $\begin{array}{l}\text { Common Mynah } \\
\text { Acridotheres tristis }\end{array}$ & $\mathrm{br}$ & + & + & & + & + & & \\
\hline $\begin{array}{l}\text { Hill Mynah } \\
\text { Gracula religiosa }\end{array}$ & $\mathrm{br}$ & & & + & & + & & + \\
\hline \multicolumn{9}{|l|}{ Family Corvidae } \\
\hline $\begin{array}{l}\text { House Crow } \\
\text { Corvus splendens }\end{array}$ & $b r$ & & & & & + & & \\
\hline $\begin{array}{l}\text { Jungle Crow } \\
\text { Corvus macrorhynchos }\end{array}$ & $\mathrm{br}$ & & & & & + & & \\
\hline \multicolumn{9}{|l|}{ Family Campephagidae } \\
\hline $\begin{array}{l}\text { Large Cuckoo-shrike } \\
\text { Coracina macei }\end{array}$ & $\mathrm{br}$ & & & + & + & + & & \\
\hline $\begin{array}{l}\text { Orange Minivet } \\
\text { Pericrocotus flammeus }\end{array}$ & $\mathrm{br}$ & & & + & + & + & & \\
\hline
\end{tabular}




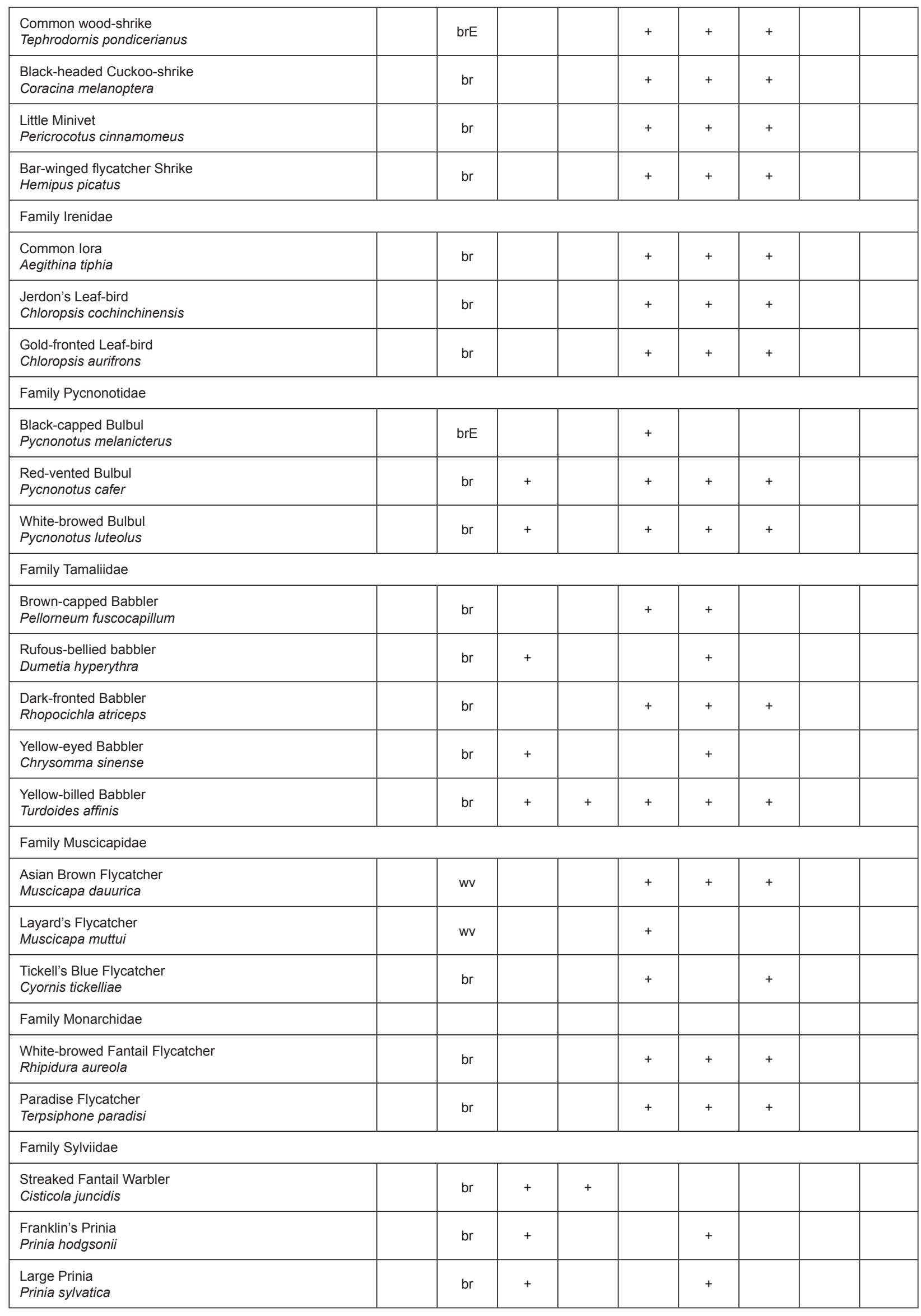




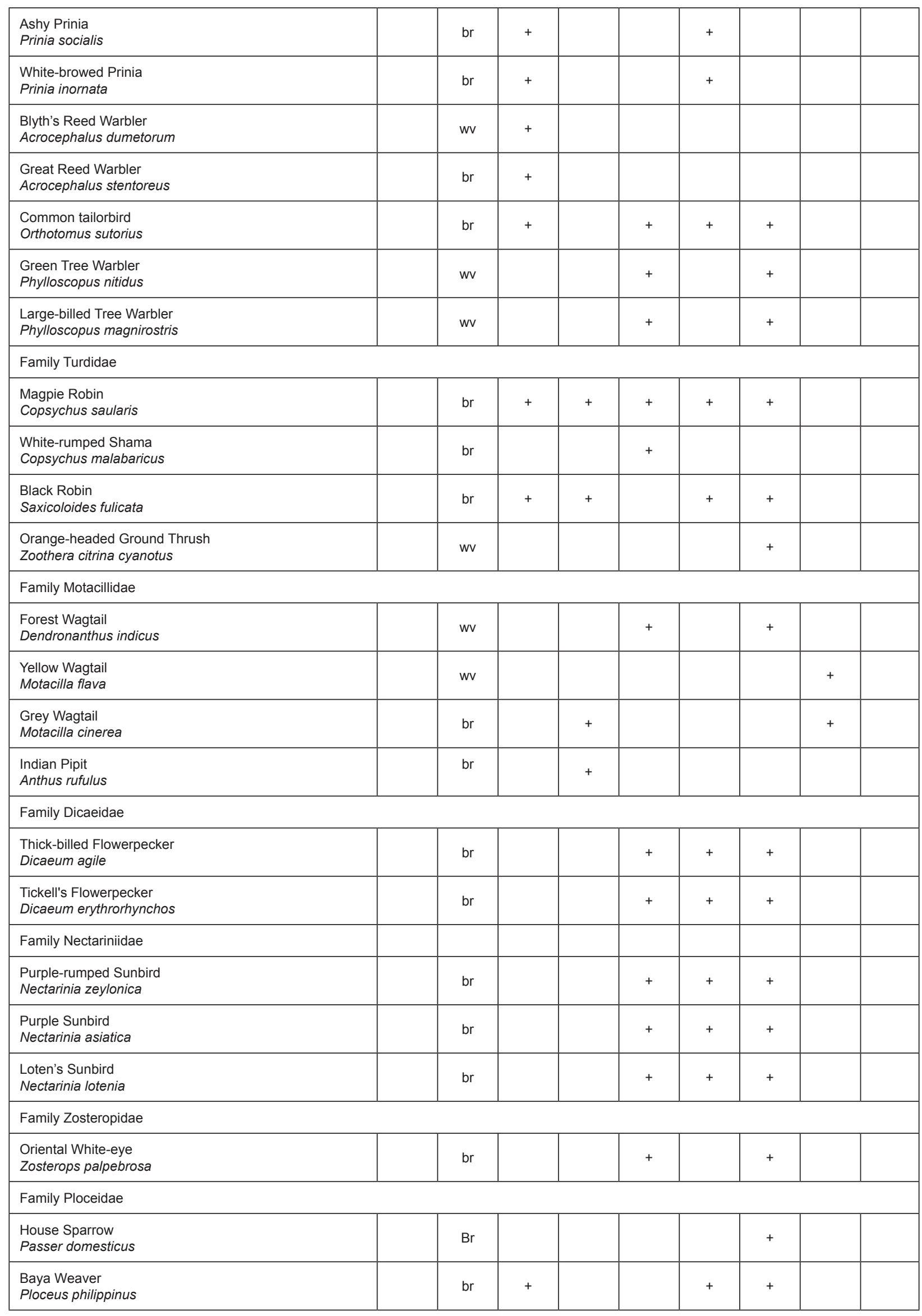




\begin{tabular}{|c|c|c|c|c|c|c|c|c|}
\hline \multicolumn{9}{|l|}{ Family Estrildidae } \\
\hline $\begin{array}{l}\text { White-backed Munia } \\
\text { Lonchura striata }\end{array}$ & $\mathrm{br}$ & + & & & + & + & & \\
\hline $\begin{array}{l}\text { Spotted Munia } \\
\text { Lonchura punctulata }\end{array}$ & $\mathrm{br}$ & + & & & + & + & & \\
\hline $\begin{array}{l}\text { Black-headed Munia } \\
\text { Lonchura malacca }\end{array}$ & $\mathrm{br}$ & + & & & + & & & \\
\hline \multicolumn{9}{|l|}{$\begin{array}{l}\text { Order Upupiformes } \\
\text { Family Upupidae }\end{array}$} \\
\hline $\begin{array}{l}\text { Common Hoopoe } \\
\text { Upupa epops }\end{array}$ & $\mathrm{br}$ & + & & & + & & & \\
\hline Total number of species in each habitat & & 45 & 28 & 68 & 78 & 99 & 18 & 20 \\
\hline
\end{tabular}

brE - Breeding resident endemic; br - Breeding resident non-endemics; wv - Winter visitor

The mosaic nature yields multiple habitat edges enriched with many microhabitat features suitable for different species (Cramer \& Willig 2005).

2. The university premises are significantly high in resource availability for the birds. For instance, several species of nectarine and fruit-bearing plant species are grown within the study site, especially in the home gardens. Further, multiple habitats provide ample niches and microhabitat conditions for invertebrates. Hence, the university premises provide ample food for different feeding guilds of birds.

3. The study site connects the adjacent forested hills; "Andampahura" and "Rahathangala" by functioning as a corridor facilitating the movement of birds. Besides, it is a habitat island that facilitates colonization of species from adjacent forests.

In this study, we noted that home gardens had the highest avifaunal diversity followed by scrub forests. However, Shahabuddin \& Kumar (2006) found that bird diversity was significantly low in disturbed habitats. This discrepancy can be explained with the intermediate disturbance hypothesis which states that species diversity is highest when disturbances are moderate in intensity and frequency (Connell 1978).

\section{Threats and conservation measures}

Despite being human-inhabited, the university premises are relatively safe from threats that endanger avifauna devoid of hunting and timber extraction pressures. We noted significant extents of habitat destruction and modification. Villagers of Buttala set fire to grasslands annually in the dry season for cattle ranching that rapidly spread to the grasslands in the campus. Uncontrolled pyrrhic events are disastrous for birds (Freckleton 2004). We noted drastic declines of grassland birds since the fires destroyed nests, eggs and hatchlings. Besides, gardeners regularly mow taller grasses using machinery which destroy the nests of many ground and undershrub nesters such as prinias, quails, munias, weavers and babblers. Mowing reduces the thickness of the grassland vegetation and makes birds and their eggs vulnerable for predation, especially by domestic cats. Although lumbering is not practiced much, felling trees for timber, construction and firewood in forest areas deter forestspecialists and allow opportunistic predators and invasive species to invade forested areas (Rosenberg 1990; Buckley et al. 2007). Seasonal and perennial pools of the site, which were used by waterfowl, were land-filled. Domestic animals such as cats were noted to extensively predate on birds (adults, eggs and young) such as yellowwattled lapwing, black robins, babblers and doves. Our frequent observations on litters size of 8-10 across years indicate increasing abundance of domestic cats. The ship rat (Rattus rattus) is rapidly colonizing this site. They predate on birds, compete with granivore birds (Yom-Tov et al. 1999) and serve as vectors for diseases (Coura et al. 2002).

With respect to the threats observed, we suggested several conservation measures. Setting fires on grasslands should be prohibited through legislation by the local government. Grasslands should be mowed less frequently. Complete mowing should be discouraged. However, the establishment of dichotomous forest species within grasslands and scrublands should be prevented to stem secondary successions. This requires intensive monitoring and eradication of seedlings of ecological "invaders". Old-grown woody tress in the grasslands may facilitate the colonization of more woody species by shading seedlings from intense solar heating. Therefore, it is important to trim the canopy of old-grown trees in the grasslands (Fischer et al. 2005).

In order to attract more core-forest avifauna such as pheasants, partridges, pigeons, doves, true owls and largely-arboreal birds, it is imperative that extraction of timber and firewood is minimized in the forested habitats (Wohlgemuth et al. 2002). Nevertheless, actively managing the abundance of dominant woody species and allowing other subordinate native forest species to establish will enhance the habitat heterogeneity of the forest habitats (Ricklefs 1977). 
Land-filling of limnotic habitats and other wetlands should be stopped. We suggest that the longevity of these habitats be ensured by preventing sedimentation through maintenance of riparian vegetation. We advise that ship rats be eradicated from the campus with non-fatal traps where non-targeted captives can be released back unharmed (Atkinson 1977). Monitoring and subsequent eradication of invasive plants is essential since human activities make the site vulnerable for invasion.

Apart from threat mitigation, we recommend several management strategies that would enhance the existing avifaunal diversity. Maintaining snags in home gardens and forest habitats will recruit more arboreal, cavity nesting birds such as parrots, owls, kingfishers, barbets and woodpeckers. Protection of wetlands from land-filling and draining will improve the on-site reproduction success of waterfowls Dickson et al. 1983). Floral diversity of home grades should be enriched via introduction of native nectarine species and fruit-bearing plants. Garden wastes should not be burnt but be disposed sanitarily, encouraging colonization of invertebrate prey. We proposed that application of synthetic pesticides in campus premises be minimized and alternatives be sought. Building up small cascades or ponds to replenish water and thermoregulation will effectively attract birds (Solecki \& Rosenzweig 2004).

Further studies should be targeted on ecology, especially reproductive biology, population dynamics and health of birds of the university premises. For efficient management and conservation of avifauna, a comprehensive knowledge on the ecology and the life histories of the birds are required. With such information, the habitats of the university premises can be managed much more appropriately to enhance the resource availability and habitat suitability for different species of birds which in turn will improve the long-term viability of a rich assemblage of avifauna in the university premises.

\section{REFERENCES}

Aston, P.S. \& C.V.S. Gunathilleke (1987a). New light on the plant geography of Ceylon I. Historical plant geography. Journal of Biologeography 14:249-285.

Aston, P.S. \& C.V.S. Gunathilleke (1987b). New light on the plant geography of Ceylon II. The ecological biogeography. Journal of Biologeography 14:295-327.

Atkinson, I.A.E. (1978). Evidence for effects of rodents on the vertebrate wildlife of New Zealand islands, pp.7-30. In: Dingwall, P.R., I.A.E. Atkinson \& C. Hay (eds.). The Ecology and Control of Rodents in New Zealand Nature Reserves, Department of Lands and Survey Information Series No 4, Wellington, New Zealand.

Connell, J.H. (1978). Diversity in tropical rain forests and coral reefs. Science 199:1302-1310.

Coura J.R., A.C.V. Junqueira, O. Fernandes, S.A.S. Valente, \& M.A. Miles (2002). emerging Chagas disease in Amazonian Brazil. Trends in Parasitology 18:171-176.
Cramer, M.J. \& M.R. Willig (2005). Habitat heterogeneity, species diversity and null models. Oikos 108:209-218.

De Alwis, C. \& T.D. Surasinghe (2006). Diversity of butterflies in the premises of Sabaragamuwa University, Buttala, Sri Lanka. International Forestry \& Environment Symposium 2006, Annual Symposium Proceedings of the Department of Forestry and Environmental Science, University of Sri Jayewardenepura, Sri Lanka.

De Alwis, C., T.D. Surasinghe \& E. Kudavidanage (2006). Diversity of birds in the premises of Sabaragamuwa University, Butthala, Sri Lanka. International Symposium on Issues and Challenges of the 21st Century, Sabaragamuwa University of Sri Lanka, Belihuloya.

Dickson, J., G.R.N. Conner \& J.H. Williamson (1983). Snag retention increases bird use of a clear-cut. Journal of Wildlife Management 47: 799-804.

Fischer, R.A., C. Eberly \& D. Keough (2005). Supporting Habitat Management for Bird Conservation Planning on Fort Belvoir, Virginia. USDA Forest Service General Technical Report 191:1178-1183.

Hietala-Koivu, R., T. Järvenpää \& J. Helenius (2004). Value of semi-natural areas as biodiversity indicators in agricultural landscapes. Agricultural Ecosystems \& Environment 101: 9-19.

IUCN (2009). IUCN Red List of Threatened Species. Version 2009.1. <www.iucnredlist.org>. Downloaded on 24 July 2009.

Kaluthota, C.D. \& S.W. Kotagama (2008). Historical changes in number of Endemic birds in Sri Lanka from 1852 to 2006. Annual Forestry and Environment Symposium, University of Sri Jayewardenepura, Sri Lanka.

Kotagama, S.W., R.I. De Silva, A.S. Wijayasinha \& V Abeygunawardena (2006). Avifaunal list of Sri Lanka, pp. 164-203. In: Bambaradeniya, C.N.B (eds.). Fauna of Sri Lanka: Status of Taxonomy, Research and Conservation. The World Conservation Union, Colombo, Sri Lanka, 308pp.

Ricklefs, R.E. (1977). Environmental heterogeneity and plant species diversity: A hypothesis. The American Naturalist 111: 376-381

Shahabuddin, G. \& R. Kumar (2006). Influence of anthropogenic disturbance on bird communities in a tropical dry forest: role of vegetation structure. Animal Conservation 9: 404-413

Solecki, W.D. \& C. Rosenzweig (2004). Biodiversity, Biosphere Reserves, and the Big Apple: A Study of the New York Metropolitan Region. Annals of the New York Academy of Sciences 1023:105-124.

Survey Department (1987). Sri Lanka 1:50,000 Topographic Quadrangle Maps. 1:50,000. Survey Department, Colombo, Sri Lanka.

Survey Department. (1988). National Atlas of Sri Lanka. Survey Department, Colombo, Sri Lanka.

Temple, S.A. (1977). The concept of managing endangered birds. pp3-8. In: Temple, S.A. (ed.). Endangered Birds: Management Techniques for Persevering Threatened Species. University of Wisconsin Press, Wisconsin, USA.

Wohlgemuth, T., M. Bürgi, C. Scheidegger \& M. Schütz (2002). Dominance reduction of species through disturbance: a proposed management principle for central Europe forests. Forest Ecology and Management 166: 1-15.

Yom-Tov, Y., S. Yom-Tov \& H. Moller (1999). Competition, coexistence, and adaptation amongst rodent invaders to Pacific and New Zealand islands. Journal of Biogeography 26: 947-958. 